\title{
Briefs
}

\section{Modeling the Fringing Electric Field Effect on the Threshold Voltage of FD SOI nMOS Devices With the LDD/Sidewall Oxide Spacer Structure}

\author{
S. C. Lin and J. B. Kuo
}

\begin{abstract}
This paper presents analysis of the fringing electric field effect on the threshold voltage of fully depleted (FD) silicon-on-insulator nMOS devices with the lightly doped drain (LDD)/sidewall oxide spacer structure based on a closed-form analytical model derived from the two-dimensional (2-D) Poisson's equation and using the conformal mapping technique. Based on the analytical model, as verified by the experimental data and the 2-D simulation results, with a lower n-LDD doping density, the fringing electric field effect in the sidewall oxide spacer lowers the short-channel effect.
\end{abstract}

Index Terms-Device model, fringing electric field, fully depleted, silicon-on-insulator (SOI), nMOS.

\section{INTRODUCTION}

Fully depleted (FD) silicon-on-insulator (SOI) CMOS devices provide advantages in reduced short-channel effects and smaller floating body effects [1], [2]. As for bulk counterparts, the LDD/sidewall oxide spacer structure has also been used in a short-channel FD SOI CMOS devices to reduce high electric field effects [3] [4]. Short-channel effects of the FD SOI CMOS devices have been reported [5], [6]. In fact, the fringing electric field in the sidewall oxide spacer is important since it may affect the performance of the FD SOI CMOS devices with the LDD/sidewall oxide spacer structure. Recently, fringing induced barrier lowering (FIBL) in a sub-100-nm nMOS device in terms of the gate dielectric constant has been reported [7]. In fact, the fringing electric field in the sidewall oxide spacer region may have a strong impact on the device performance. Due to the complexity from the LDD/sidewall oxide spacer structure, until now no analytical model for the fringing electric field related threshold voltage is available. In this paper, the analysis of the fringing electric field effect of FD SOI nMOS devices with the LDD/sidewall oxide spacer structure based on a closed-form analytical model derived from the two-dimensional (2-D) Poisson's equation and using the conformal mapping technique is described. It will be shown that based on the analytical model, as verified by the experimental data and the 2-D simulation results, with a lower n-LDD doping density, the fringing electric field effect in the sidewall oxide spacer lowers the short-channel effect.

\section{MODEL DERIVATION}

In order to concentrate on the fringing electric field effect and to simplify the analysis, an FD SOI nMOS device with an LDD/sidewall oxide spacer structure has been studied [3]. Fig. 1 shows the 2-D electric field contours in a $0.25-\mu \mathrm{m}$ FD SOI nMOS device with a $0.1-\mu \mathrm{m}$ lightly doped drain LDD/sidewall oxide spacer structure and an $\mathrm{n}^{+}$ polysilicon gate of $2000 \AA$, biased at $V_{\mathrm{DS}}=0.1 \mathrm{~V}$ and the threshold

Manuscript received May 13, 2003; revised July 16, 2003. The review of this brief was arranged by Editor J. Vasi.

The authors are with the Department of Electrical Engineering, National Taiwan University, Taipei, Taiwan 106-17, R.O.C. (e-mail jbkuo@cc.ee.ntu.edu.tw).

Digital Object Identifier 10.1109/TED.2003.816910

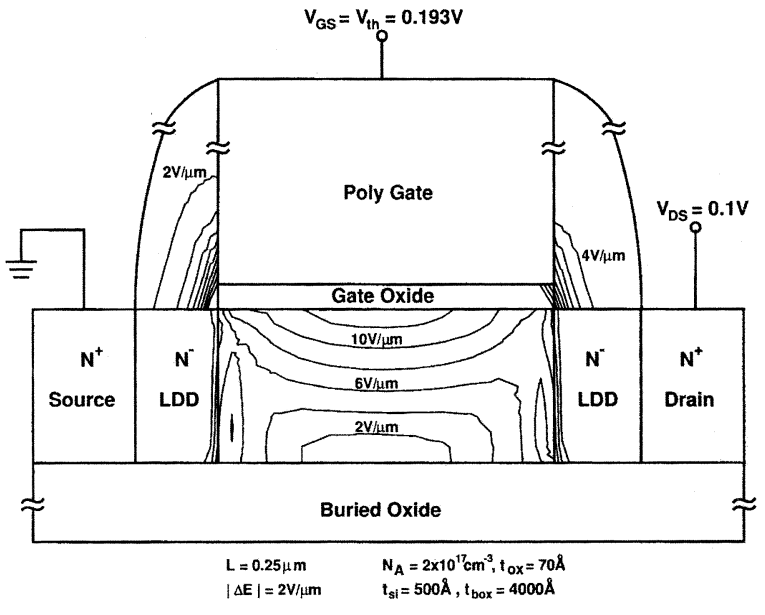

Fig. 1. Two-dimensional electric field contours in a $0.25-\mu \mathrm{m}$ FD SOI nMOS device with a $0.1 \mu \mathrm{m}$ lightly doped drain (LDD)/sidewall oxide spacer structure, having an $\mathrm{n}^{+}$polysilicon gate of $2000 \AA$, a front gate oxide of $70 \AA$, a thin-film of $500 \AA$ A doped with a p-type doping density of $2 \times 10^{17} \mathrm{~cm}^{-3}$, and a buried oxide of $4000 \AA$, biased at $V_{\mathrm{DS}}=0.1 \mathrm{~V}$ and the threshold voltage $-V_{\mathrm{GS}}=0.193 \mathrm{~V}$.

voltage $-V_{\mathrm{GS}}=0.193 \mathrm{~V}$, based on 2-D simulation results [9]. As shown in the figure, the n-LDD region is slightly depleted near the pn junction close to the $\mathrm{p}$-channel region. In the sidewall oxide spacer region, fringing electric field contours from the polysilicon gate to the $\mathrm{n}$-LDD region can be seen. In addition, these fringing electric field contours are most noticeable near the corner of the polysilicon gate and $n-L D D$ region. From the 2-D electric field contours, the fringing electric field effect in the sidewall oxide spacer may influence the operation of the device via the depleted n-LDD region.

For the FD SOI nMOS device under study [Fig. 2(a)], the origin is at the source end of the channel under the front gate oxide. The $x$ axis is in the substrate direction and the $y$ axis is in the lateral channel direction toward the drain. A quasi-2-D approach as used in modeling the partially depleted SOI devices [10] has been adopted to solve the 2-D Poisson's equation. In order to facilitate model derivation, the depletion region in the thin-film of the device is divided into three portions:

1) the n-LDD depletion region near the source (I);

2) the n-LDD depletion region near the drain (II);

3) the p-channel region under the gate (III).

In region (I), the $\mathrm{n}-\mathrm{LDD}$ depletion region near the source, its width is determined by the doping density of the p-type channel region $\left(N_{A}\right)$ and the n-LDD region $\left(N_{D}\right)$ as

$$
L_{s}=\sqrt{\frac{2 \epsilon_{\mathrm{si}}}{\mathrm{q}} N_{A} /\left(N_{A}+N_{D}\right) \frac{V_{\mathrm{bi}}}{\mathrm{N}_{\mathrm{D}}}}
$$

where $V_{\mathrm{bi}}$ is the built-in voltage:

$$
V_{\mathrm{bi}}=\frac{\mathrm{kT}}{\mathrm{q}} \ln \left(\frac{N_{D} N_{A}}{n_{i}^{2}}\right)
$$

$\mathrm{n}_{\mathrm{i}}$ is the intrinsic concentration, $\epsilon_{\mathrm{si}}$ is silicon permittivity, and $\mathrm{q}$ is the electron charge. In region (II), the n-LDD depletion region near the drain, its width is decided by the drain voltage as

$$
L_{d}=\sqrt{\frac{2 \epsilon_{\mathrm{si}}}{\mathrm{q}} \frac{N_{A}}{\left(N_{A}+N_{D}\right)} \frac{\left(V_{\mathrm{bi}}+V_{\mathrm{DS}}\right)}{\mathrm{N}_{\mathrm{D}}}} .
$$

Note that in $L_{s}$ and $L_{d}$, the 2-D effect has been neglected. 


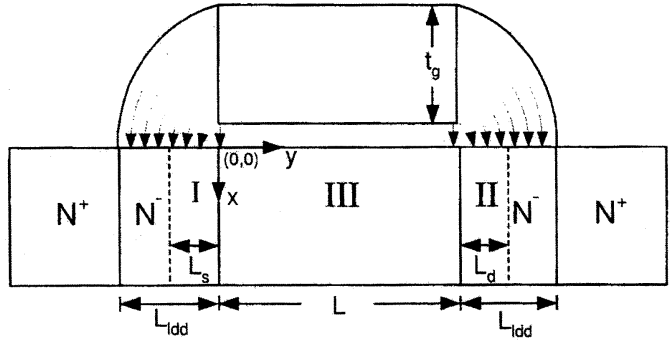

(a)

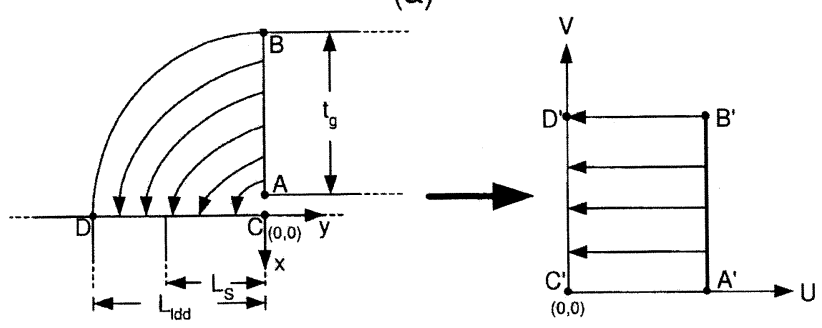

(b)

Fig. 2. Cross section of the sidewall oxide spacer in the FD SOI nMOS showing the conformal mapping technique.

In these three depleted regions in the thin-film, the electrostatic potential can be approximated using the polynomial as shown below [8]

$$
\Psi_{i}(x, y)=a_{0 i}(y)+a_{1 i}(y) x+a_{2 i}(y) x^{2}
$$

where $i$ is (1), (2), or (3), representing the three depleted regions, respectively. Equation (1) should satisfy the 2-D Poisson's equation

$$
\frac{\partial^{2} \Psi_{i}(x, y)}{\partial x^{2}}+\frac{\partial^{2} \Psi_{i}(x, y)}{\partial y^{2}}=-\frac{q N}{\epsilon_{\mathrm{si}}}
$$

where $N$ is equal to $N_{D}$ in the n-LDD region and $-N_{A}$ in the p-type channel region. The four boundary conditions for the 2-D Poisson's equation are described as follows: 1) at the front gate oxide/thin-film interface $(x=0)$, the electrostatic potential is $\Psi_{i}(0, y)=\Psi_{\mathrm{sfi}}(y)$, where $\Psi_{\mathrm{sfi}}(y)$ is the surface electrostatic potential of the thin-film at location $y$. 2) At the buried oxide/thin-film interface $\left(x=t_{\mathrm{si}}\right)$, where $t_{\mathrm{si}}$ is the thin-film thickness, the electrostatic potential is $\Psi_{i}\left(t_{\mathrm{si}}, y\right)=$ $\Psi_{\text {sbi }}(y)$, where $\Psi_{\text {sbi }}(y)$ is the electrostatic potential on the top of the buried oxide. 3) At the front gate oxide/thin-film interface $(x=0)$, from Gauss Law, the displacement is continuous. Thus, one obtains

$$
\left.\frac{\partial \Psi_{i}(x, y)}{\partial x}\right|_{x=0}=-\frac{\epsilon_{\mathrm{ox}}}{\epsilon_{\mathrm{si}}} \frac{\Psi_{\mathrm{Gf}}-\Psi_{s f i}(y)}{t(y)}
$$

where $\epsilon_{\mathrm{ox}}$ is oxide permittivity, $\Psi_{\mathrm{Gf}}$ is the electrostatic potential of the polysilicon gate $\Psi_{\mathrm{Gf}}=V_{G}-\phi_{f g}, \phi_{\mathrm{fg}}=(\mathrm{kT} / \mathrm{q}) \ln \left(\mathrm{N}_{\mathrm{D}} / \mathrm{n}_{\mathrm{i}}\right)$ is the fermi potential of the n-type polysilicon gate, and $t(y)$ is the distance between the gate electrode and the thin-film/front oxide interface. 4) Similarly, at the buried oxide/thin-film interface, from Gauss Law, one obtains

$$
\left.\frac{\partial \Psi_{i}(x, y)}{\partial x}\right|_{x=t_{\mathrm{si}}}=-\frac{\epsilon_{\mathrm{ox}}}{\epsilon_{\mathrm{si}}} \frac{\Psi_{\mathrm{sbi}}(y)-\Psi_{\mathrm{Gb}}}{t_{\mathrm{box}}}
$$

where $t_{\mathrm{box}}$ is the thickness of the buried oxide and $\Psi_{\mathrm{Gb}}$ is the electrostatic potential of the back gate $\left(\Psi_{\mathrm{Gb}}=V_{\mathrm{sub}}-\phi_{\mathrm{fsub}}\right), V_{\mathrm{sub}}$ is the back gate bias, and $\phi_{\mathrm{fsub}}=(\mathrm{kT} / \mathrm{q}) \ln \left(\mathrm{n}_{\mathrm{i}} / \mathrm{N}_{\mathrm{sub}}\right)$ is the fermi potential of the p-type substrate.

\section{A. Region (I)}

In region (I) - in the n-LDD depletion region under the sidewall oxide spacer near the source, it is affected by the fringing electric field in the sidewall oxide spacer. A conformal mapping technique [8], [11] has been applied to simplify the analysis. In region (I), from (1) and the boundary conditions, the electrostatic potential at the thin-film/buried oxide interface and the coefficients in the electrostatic potential $\Psi_{1}(x, y)$ are

$$
\begin{aligned}
\Psi_{\mathrm{sb} 1}(y)=\frac{\epsilon_{\mathrm{si}}}{2 C_{s}+C_{\mathrm{box}}}\left\{-\frac{\epsilon_{\mathrm{ox}}}{\epsilon_{\mathrm{si}}}\right. & \frac{\Psi_{\mathrm{Gf}}-\Psi_{\mathrm{sf} 1}(y)}{t(y)} \\
& \left.+\frac{2}{t_{\mathrm{si}}} \Psi_{\mathrm{sf} 1}(y)+\frac{C_{\mathrm{box}}}{\epsilon_{\mathrm{si}}} \Psi_{\mathrm{Gb}}\right\}
\end{aligned}
$$

where

$$
\begin{gathered}
a_{01}(y)=\Psi_{\mathrm{sf} 1}(y), \quad a_{11}(y)=-\frac{\epsilon_{\mathrm{ox}}}{\epsilon_{\mathrm{si}}} \frac{\Psi_{\mathrm{Gf}}-\Psi_{\mathrm{sf} 1}(y)}{t(y)} \\
a_{21}(y)=\frac{1}{t_{\mathrm{si}}^{2}}\left\{\Psi_{\mathrm{sb} 1}(y)-\left[1+\frac{\epsilon_{\mathrm{ox}}}{\epsilon_{\mathrm{si}}} \frac{t_{\mathrm{si}}}{t(y)}\right] \Psi_{\mathrm{sf} 1}(y)\right. \\
\left.+\frac{\epsilon_{\mathrm{ox}}}{\epsilon_{\mathrm{si}}} \frac{t_{\mathrm{si}}}{t(y)} \Psi_{\mathrm{Gf}}\right\} .
\end{gathered}
$$

In order to simplify the effect of the nonfixed value of the distance $(t(y))$ between the gate electrode and the thin-film/front oxide interface on the term $\Psi_{\mathrm{Gf}}-\Psi_{\mathrm{sf} 1}(y) / t(y)$ in $a_{11}(y)$, in the n-LDD region under the sidewall oxide spacer, conformal mapping transformation technique [8], [11] has been used to transform the original $x \vec{X}+y \vec{Y}$ space in terms of the $\vec{X}$ and $\vec{Y}$ axes as shown in Fig. 3(b) into the $u \vec{U}+V \vec{V}$ space in terms of $\vec{U}$ and $\vec{V}$ axes based on the following transfer function: $y \vec{Y}+n x \vec{X}=K \sin (u \vec{U}+v \vec{V})$, where

$$
\begin{gathered}
n=\frac{L_{\mathrm{ldd}}}{t_{\mathrm{ox}} \sinh \left[\cosh ^{-1}\left(\frac{t_{\mathrm{ox}}+t_{g}}{t_{\mathrm{ox}}}\right)\right]} \\
K=\frac{-L_{\mathrm{ldd}}}{\sinh \left[\cosh ^{-1}\left(\frac{t_{\mathrm{ox}}+t_{g}}{t_{\mathrm{ox}}}\right)\right]}
\end{gathered}
$$

and $L_{\mathrm{ldd}}$ is the width of the n-LDD region. Based on the above formula, $\mathrm{ABCD}$ in the $x \vec{X}+y \vec{Y}$ coordinates is transformed into $\mathrm{A}^{\prime} \mathrm{B}^{\prime} \mathrm{C}^{\prime} \mathrm{D}^{\prime}$ in the $u \vec{U}+V \vec{V}$ coordinates. Under this condition, the arc-shaped electric field contour in the sidewall oxide spacer in the $x \vec{X}+y \vec{Y}$ coordinates has become straight-line-shaped in the $u \vec{U}+V \vec{V}$ coordinates. Based on this transformation, the distance between the gate electrode and the n-LDD/sidewall oxide spacer interface $t(y)$, which is not a fixed value, has been transformed into $m \pi / 2$, which is the distance between points $\mathrm{A}^{\prime}$ and $\mathrm{C}^{\prime}$ in the new coordinates, with the condition that $m$ must satisfy $\sin (m \pi / 2)=1$. Based on the conformal mapping formula, the term- $-\Psi_{\mathrm{Gf}}-\Psi_{\mathrm{sf} 1}(y) / t(y)$ has been simplified to $\left(\Psi_{\mathrm{Gf}}-\Psi_{v}(v)\right) /(m \pi / 2)$ in the new coordinates. Therefore, one also obtains: $y=K \sinh V$ at the thin-film/front gate oxide interface $(x=0)$. With the transformation, one obtains

$$
\begin{aligned}
& a_{01}(v)=\Psi_{v}(v) \\
& a_{11}(v)=-\frac{\epsilon_{\mathrm{ox}}}{\epsilon_{\mathrm{si}}} \frac{\Psi_{\mathrm{Gf}}-\Psi_{v}(v)}{\frac{m \pi}{2}} \\
& a_{21}(v)=\alpha_{0} \Psi_{v}(v)+\beta_{0}
\end{aligned}
$$

where

$$
\alpha_{0}=\frac{1}{t_{\mathrm{si}}^{2}}\left\{-\frac{C_{s}+C_{\mathrm{box}}}{\left(2 C_{s}+C_{\mathrm{box}}\right) C_{s}} \frac{2 \epsilon_{\mathrm{ox}}}{m \pi}-\frac{C_{\mathrm{box}}}{2 C_{s}+C_{\mathrm{box}}}\right\}
$$

and

$$
\beta_{0}=\frac{1}{t_{\mathrm{si}}^{2}}\left\{\frac{C_{s}+C_{\mathrm{box}}}{\left(2 C_{s}+C_{\mathrm{box}}\right) C_{s}} \frac{2 \epsilon_{\mathrm{ox}}}{m \pi} \Psi_{\mathrm{Gf}}+\frac{C_{\mathrm{box}}}{2 C_{s}+C_{\mathrm{box}}} \Psi_{\mathrm{Gb}}\right\} .
$$

The 2-D Poisson's equation of (2) can be further simplified using the following approach [12]—at any depth $(x)$ in the thin-film, the deriva- 
tive of the electric field in the lateral channel direction is related to the derivative of the surface electric field as follows:

$$
\frac{\partial^{2} \Psi_{i}(x, y)}{\partial y^{2}}=\frac{1}{k_{s}} \frac{d^{2} \Psi_{s f i}(y)}{d y^{2}}
$$

where $k_{s}$ is a parameter less than $1\left(k_{s}<1\right)$. In region (I), from (5) with the conforming mapping technique, one obtains

$$
\begin{aligned}
& \frac{\partial^{2} \Psi_{1}(x, y)}{\partial y^{2}}=\frac{1}{k_{s}}\left[\frac{d^{2} \Psi_{v}(v)}{d v^{2}} \frac{1}{K^{2} \cosh ^{2} v}\right. \\
& \left.\quad-\frac{d \Psi_{v}(v)}{d v} \frac{\sinh v}{K^{2} \cosh ^{3} v}\right]
\end{aligned}
$$

in the new coordinates. Therefore, a second-order differential equation in terms of the surface electrostatic potential in the new coordinates has been obtained

$$
\begin{aligned}
& \frac{d^{2} \Psi_{v}(v)}{d v^{2}}-(\tanh v) \frac{d \Psi_{v}(v)}{d v}+\left(B_{0} \cosh ^{2} v\right) \Psi_{v}(v) \\
& \quad=B_{1} \cosh ^{2} v \\
& B_{0}=\alpha_{0} k_{s} K^{2} \\
& B_{1}=-\left(\frac{q N_{D}}{\epsilon_{\mathrm{si}}}+\beta_{0}\right) k_{s} K^{2} .
\end{aligned}
$$

Solving (7), the surface electrostatic potential in the new coordinates is

$$
\begin{aligned}
\Psi_{v}(v) & =g_{1} e^{r \sinh v}+g_{2} e^{-r \sinh v}+\frac{B_{1}}{B_{0}} \\
r & =\sqrt{-B_{0}} .
\end{aligned}
$$

Using the conformal mapping transformation $(y=K \sinh V)$, (8) becomes

$$
\Psi_{\mathrm{sf} 1}(y)=g_{1} e^{(r / K) y}+g_{2} e^{-(r / K) y}+\frac{B_{1}}{B_{0}}
$$

in the original coordinates. Considering the boundary conditions: 1) at the left end of the n-LDD depletion region, the surface electrostatic potential is equal to $\Psi_{\mathrm{sf} 1}\left(-L_{s}\right)=\phi_{\mathrm{fn}}$, where $\phi_{\mathrm{fn}}=(\mathrm{kT} / \mathrm{q}) \ln \left(\mathrm{N}_{\mathrm{D}} / \mathrm{n}_{\mathrm{i}}\right)$ and $N_{D}$ is the doping density of the n-LDD region; 2 ) at the right end, the surface electrostatic potential is equal to $\Psi_{\text {sf } 1}(0)=\Psi_{1}$, then the surface electrostatic potential in region (I) becomes

$$
\Psi_{\mathrm{sf} 1}(y)=g_{1} e^{(r / K) y}+g_{2} e^{-(r / K) y}+\frac{B_{1}}{B_{0}}
$$

where

$$
\begin{aligned}
g_{1}= & \frac{1}{2 \sinh \left(\frac{r}{K} L_{s}\right)}\left\{-\left(\phi_{f n}-\frac{B_{1}}{B_{0}}\right)\right. \\
& \left.+\left(\Psi_{1}-\frac{B_{1}}{B_{0}}\right) e^{(r / K) L_{s}}\right\}
\end{aligned}
$$

and

$$
\begin{aligned}
g_{2}= & \frac{1}{2 \sinh \left(\frac{r}{K} L_{s}\right)}\left\{\left(\phi_{f n}-\frac{B_{1}}{B_{0}}\right)\right. \\
& \left.-\left(\Psi_{1}-\frac{B_{1}}{B_{0}}\right) e^{-(r / K) L_{s}}\right\} .
\end{aligned}
$$

\section{B. Region (II)}

In region (II), its structure is identical to that in region (I). Therefore, the approach in obtaining the electrostatic potential $\left(\Psi_{2}(x, y)\right)$ and the surface electrostatic potential $\left(\Psi_{\mathrm{sf} 2}(y)\right)$ is similar as for region (I) except the boundary conditions: 1 ) at the left end of the depleted n-LDD region, its surface electrostatic potential is $\Psi_{\mathrm{sf} 2}(L)=\Psi_{2}$ and 2) at the right end, it is $\Psi_{\mathrm{sf} 2}\left(L+L_{d}\right)=V_{\mathrm{DS}}+\phi_{\mathrm{fn}}$. Consequently, the surface electrostatic potential in region (II) is

$$
\Psi_{s f 2}(y)=g_{3} e^{-(r / K)(y-L)}+g_{4} e^{(r / K)(y-L)}+\frac{B_{1}}{B_{0}}
$$

where

$$
\begin{aligned}
g_{3}= & \frac{1}{2 \sinh \left(\frac{r}{K} L_{d}\right)}\left\{-\left(V_{\mathrm{DS}}+\phi_{f n}-\frac{B_{1}}{B_{0}}\right)\right. \\
& \left.+\left(\Psi_{2}-\frac{B_{1}}{B_{0}}\right) e^{(r / K) L_{d}}\right\}
\end{aligned}
$$

and

$$
\begin{aligned}
g_{4}= & \frac{1}{2 \sinh \left(\frac{r}{K} L_{d}\right)}\left\{\left(V_{\mathrm{DS}}+\phi_{f n}-\frac{B_{1}}{B_{0}}\right)\right. \\
& \left.-\left(\Psi_{2}-\frac{B_{1}}{B_{0}}\right) e^{-(r / K) L_{d}}\right\} .
\end{aligned}
$$

\section{Region (III)}

In region (III), for the p-channel depletion region under the front gate oxide, the equations derived before are still applicable except that $t(y)$ should be replaced by the front gate oxide thickness $t_{\mathrm{ox}}$. From (1), the relationship between the front surface electrostatic potential $\Psi_{\mathrm{sf} 3}(y)$ and the back surface electrostatic potential $\Psi_{\mathrm{sb} 3}(y)$, and the coefficients- $a_{03}(y), a_{13}(y)$, and $a_{23}(y)$ have been obtained:

$$
\begin{aligned}
\Psi_{s b 3}(y)=\frac{2 C_{s}+C_{\mathrm{ox}}}{2 C_{s}+C_{\mathrm{box}}} \Psi_{\mathrm{sf} 3}(y)-\frac{C_{\mathrm{ox}}}{2 C_{s}}+C_{\mathrm{box}} \Psi_{\mathrm{Gf}} \\
-\frac{C_{\mathrm{box}}}{2 C_{s}+C_{\mathrm{box}}} \Psi_{\mathrm{Gb}}
\end{aligned}
$$

where

$$
\begin{aligned}
& a_{03}(y)=\Psi_{\mathrm{sf} 3}(y) \\
& a_{13}(y)=-\frac{C_{\mathrm{ox}}}{\epsilon_{\mathrm{si}}}\left[\Psi_{\mathrm{Gf}}-\Psi_{\mathrm{sf} 3}(y)\right]
\end{aligned}
$$

and

$$
\begin{aligned}
a_{23}(y)= & \frac{1}{t_{\mathrm{si}}^{2}}\left\{-\frac{C_{\mathrm{ox}} C_{\mathrm{box}}+C_{\mathrm{ox}} C_{s}+C_{\mathrm{box}} C_{s}}{\left(2 C_{s}+C_{\mathrm{box}}\right) C_{s}} \Psi_{\mathrm{sf} 3}(y)\right. \\
& \left.+\frac{C_{\mathrm{ox}}\left(C_{\mathrm{box}}+C_{s}\right)}{\left(2 C_{s}+C_{\mathrm{box}}\right) C_{s}} \Psi_{\mathrm{Gf}}-\frac{C_{\mathrm{box}}}{2 C_{s}+C_{\mathrm{box}}} \Psi_{\mathrm{Gb}}\right\} .
\end{aligned}
$$

From (12), one obtains a second-order differential equation in terms of the surface electrostatic potential

$$
\frac{d^{2} \Psi_{\mathrm{sf} 3}(y)}{d y^{2}}-r_{1}^{2} \Psi_{\mathrm{sf} 3}(y)=G_{1}
$$

where

$$
\begin{aligned}
G_{1}= & \frac{q N_{A} k_{s}}{\epsilon_{\mathrm{si}}}-\frac{2 k_{s}}{t_{\mathrm{si}}^{2}} \frac{C_{\mathrm{ox}} C_{s}+C_{\mathrm{box}} C_{s}}{\left(2 C_{s}+C_{\mathrm{box}}\right) C_{s}} \Psi_{\mathrm{Gf}} \\
& +\frac{2 k_{s}}{t_{\mathrm{si}}^{2}} \frac{C_{\mathrm{box}}}{2 C_{s}+C_{\mathrm{box}}} \Psi_{\mathrm{Gb}} \\
= & G_{00}+G_{02} \Psi_{\mathrm{Gf}}+G_{03} \Psi_{\mathrm{Gb}}
\end{aligned}
$$

and

$$
r_{1}=\sqrt{\frac{2 k_{s}}{t_{\mathrm{si}}^{2}} \frac{C_{\mathrm{ox}} C_{s}+C_{\mathrm{box}} C_{s}+C_{\mathrm{ox}} C_{\mathrm{box}}}{\left(2 C_{s}+C_{\mathrm{box}}\right) C_{s}}} .
$$

The boundary conditions for the above equation are 1) at the left end, its surface electrostatic potential is $\Psi_{\mathrm{sf} 3}(0)=\Psi_{1}$ and 2) at the right end, it is $\Psi_{\mathrm{sf} 3}(L)=\Psi_{2}$. Solving the differential equation [(13)] with the boundary conditions, one obtains

$$
\Psi_{\mathrm{sf} 3}(y)=g_{5} e^{r_{1} y}+g_{6} e^{-r_{1} y}-\frac{G_{1}}{r_{1}^{2}}
$$


where

and

$$
g_{5}=\frac{1}{2 \sinh \left(r_{1} L\right)}\left[-\Psi_{1} e^{-r_{1} L}+\frac{G_{1}}{r_{1}^{2}}\left(1-e^{-r_{1} L}\right)+\Psi_{2}\right]
$$

$$
g_{6}=\frac{1}{2 \sinh \left(r_{1} L\right)}\left[\Psi_{1} e^{r_{1} L}-\frac{G_{1}}{r_{1}^{2}}\left(1-e^{r_{1} L}\right)-\Psi_{2}\right] .
$$

At the boundary between regions (I) and (III), the electric field should be continuous

$$
\left.\frac{\mathrm{d} \Psi_{\mathrm{sf} 1}(y)}{\mathrm{dy}}\right|_{y=0}=\left.\frac{\mathrm{d} \Psi_{\mathrm{sf} 3}(y)}{\mathrm{dy}}\right|_{y=0} .
$$

Similary, at the boundary between regions II and III, the electric field should be continuous

$$
\left.\frac{\mathrm{d} \Psi_{\mathrm{sf} 2}(y)}{\mathrm{dy}}\right|_{y=L}=\left.\frac{\mathrm{d} \Psi_{\mathrm{sf} 3}(y)}{\mathrm{dy}}\right|_{y=L} .
$$

Therefore, from the continuity of the electric field at the boundaries, the surface electrostatic potential at two ends of region (III) can be expressed

$$
\begin{aligned}
& \Psi_{1}=H_{0}+H_{1} \Psi_{\mathrm{Gf}}+H_{2} \Psi_{\mathrm{Gb}} \\
& \Psi_{2}=H_{3}+H_{4} \Psi_{\mathrm{Gf}}+H_{5} \Psi_{\mathrm{Gb}}
\end{aligned}
$$

where

$$
\begin{aligned}
& H_{0}=\frac{1}{\Omega}\left\{\left[r_{1} \operatorname{coth}\left(r_{1} L\right)+\frac{r}{K} \operatorname{coth}\left(\frac{r}{K} L_{d}\right)\right] V_{00}\right. \\
& \left.+V_{10} \frac{r_{1}}{\sinh \left(r_{1} L\right)}\right\} \\
& H_{1}=\frac{1}{\Omega}\left\{\left[r_{1} \operatorname{coth}\left(r_{1} L\right)+\frac{r}{K} \operatorname{coth}\left(\frac{r}{K} L_{d}\right)\right] V_{01}\right. \\
& \left.+V_{11} \frac{r_{1}}{\sinh \left(r_{1} L\right)}\right\} \\
& H_{2}=\frac{1}{\Omega}\left\{\left[r_{1} \operatorname{coth}\left(r_{1} L\right)+\frac{r}{K} \operatorname{coth}\left(\frac{r}{K} L_{d}\right)\right] V_{02}\right. \\
& \left.+V_{12} \frac{r_{1}}{\sinh \left(r_{1} L\right)}\right\} \\
& H_{3}=\frac{1}{\Omega}\left\{\left[r_{1} \operatorname{coth}\left(r_{1} L\right)+\frac{r}{K} \operatorname{coth}\left(\frac{r}{K} L_{s}\right)\right] V_{10}\right. \\
& \left.+V_{00} \frac{r_{1}}{\sinh \left(r_{1} L\right)}\right\} \\
& H_{4}=\frac{1}{\Omega}\left\{\left[r_{1} \operatorname{coth}\left(r_{1} L\right)+\frac{r}{K} \operatorname{coth}\left(\frac{r}{K} L_{s}\right)\right] V_{11}\right. \\
& \left.+V_{01} \frac{r_{1}}{\sinh \left(r_{1} L\right)}\right\} \\
& H_{5}=\frac{1}{\Omega}\left\{\left[r_{1} \operatorname{coth}\left(r_{1} L\right)+\frac{r}{K} \operatorname{coth}\left(\frac{r}{K} L_{s}\right)\right] V_{12}\right. \\
& \left.+V_{02} \frac{r_{1}}{\sinh \left(r_{1} L\right)}\right\} \\
& \Omega=\left[r_{1} \operatorname{coth}\left(r_{1} L\right)+\frac{r}{K} \operatorname{coth}\left(\frac{r}{K} L_{d}\right)\right] \\
& \cdot\left[r_{1} \operatorname{coth}\left(r_{1} L\right)+\frac{r}{K} \operatorname{coth}\left(\frac{r}{K} L_{s}\right)\right] \\
& -\left[\frac{r_{1}}{\sinh \left(r_{1} L\right)}\right]^{2} \\
& V_{00}=\frac{1-\cosh \left(r_{1} L\right)}{\sinh \left(r_{1} L\right)} \frac{G_{00}}{r_{1}}+\frac{\frac{r}{K}}{\sinh \left(\frac{r}{K} L_{s}\right)} \\
& \cdot\left\{\left[1-\cosh \left(\frac{r}{K} L_{s}\right)\right] \frac{q N_{D}}{\alpha_{0} \epsilon_{\mathrm{si}}}+\phi_{f n}\right\}
\end{aligned}
$$

$$
\begin{aligned}
V_{01}= & \frac{1-\cosh \left(r_{1} L\right)}{\sinh \left(r_{1} L\right)} \frac{G_{02}}{r_{1}}+\frac{\frac{r}{K}}{\sinh \left(\frac{r}{K} L_{s}\right)} \\
& \cdot\left[1-\cosh \left(\frac{r}{K} L_{s}\right)\right] \frac{2}{\alpha_{0}} \frac{\epsilon_{\mathrm{ox}}}{t_{\mathrm{si}}^{2}} \frac{2}{m \pi} \frac{C_{s}+C_{\mathrm{box}}}{\left(2 C_{s}+C_{\mathrm{box}}\right) C_{s}} \\
V_{02}= & \frac{1-\cosh \left(r_{1} L\right)}{\sinh \left(r_{1} L\right)} \frac{G_{03}}{r_{1}}+\frac{\frac{r}{K}}{\sinh \left(\frac{r}{K} L_{s}\right)} \\
& \cdot\left[1-\cosh \left(\frac{r}{K} L_{s}\right)\right] \frac{2}{\alpha_{0}} \frac{1}{t_{\mathrm{si}}^{2}} \frac{C_{\mathrm{box}}}{2 C_{s}+C_{\mathrm{box}}} \\
V_{10}= & \frac{1-\cosh \left(r_{1} L\right)}{\sinh \left(r_{1} L\right)} \frac{G_{00}}{r_{1}}+\frac{\frac{r}{K}}{\sinh \left(\frac{r}{K} L_{d}\right)} \\
& \cdot\left\{\left[1-\cosh \left(\frac{r}{K} L_{d}\right)\right] \frac{q N_{D}}{\alpha_{0} \epsilon_{\mathrm{si}}}+\left(V_{\mathrm{DS}}+\phi_{f n}\right)\right\} \\
V_{11}= & \frac{1-\cosh \left(r_{1} L\right)}{\sinh \left(r_{1} L\right)} \frac{G_{02}}{r_{1}}+\frac{\frac{r}{K}}{\sinh \left(\frac{r}{K} L_{d}\right)} \\
& \cdot\left[1-\cosh \left(\frac{r}{K} L_{d}\right)\right] \frac{2}{\alpha_{0}} \frac{\epsilon_{\mathrm{ox}}}{t_{\mathrm{si}}^{2}} \frac{2}{m \pi} \frac{C_{s}+C_{\mathrm{box}}}{\left(2 C_{s}+C_{\mathrm{box}}\right) C_{s}} \\
V_{12}= & \frac{1-\cosh \left(r_{1} L\right)}{\sinh \left(r_{1} L\right)} \frac{G_{03}}{r_{1}}+\frac{\frac{r}{K}}{\sinh \left(\frac{r}{K} L_{d}\right)} \\
& \cdot\left[1-\cosh \left(\frac{r}{K} L_{d}\right)\right] \frac{2}{\alpha_{0}} \frac{1}{t_{\mathrm{si}}^{2}} \frac{C_{\mathrm{box}}}{2 C_{s}+C_{\mathrm{box}}} .
\end{aligned}
$$

\section{Threshold Voltage}

For an FD SOI nMOS device, its threshold voltage is defined as the gate voltage when the minimum surface electrostatic potential reaches $\Psi_{\mathrm{sf} 3 \min }=\phi_{\mathrm{fp}}$, where $\phi_{\mathrm{fp}}=(\mathrm{kT} / \mathrm{q}) \ln \left(\mathrm{n}_{\mathrm{i}} / \mathrm{N}_{\mathrm{A}}\right)$ and $\mathrm{N}_{\mathrm{A}}$ is the doping denisty of the p-type thin-film. Therefore, the minimum surface electrostatic potential is $\Psi_{\mathrm{sf} 3 \mathrm{~min}}=2 \sqrt{\mathrm{g}_{5} \mathrm{~g}_{6}}-\left(\mathrm{G}_{1} / \mathrm{r}_{1}^{2}\right)$, which is equal to $\phi_{\mathrm{fp}}$ at the threshold voltage. Consequently, the threshold voltage is

$$
V_{t h}=\frac{-L_{1}+\sqrt{L_{1}^{2}-4 L_{0} L_{2}}}{2 L_{0}}+\phi_{f g}
$$

where

$$
\begin{aligned}
L_{0}= & 4 W_{1} W_{4}-\frac{G_{02}^{2}}{r_{1}^{4}} \\
L_{1}= & 4\left(W_{0} W_{4}+W_{1} W_{3}\right)-\frac{2 G_{02}}{r_{1}^{2}}-\frac{2 G_{00} G_{02}}{r_{1}^{4}} \\
& +\Psi_{\mathrm{Gb}}\left[4\left(W_{2} W_{4}+W_{1} W_{5}\right)-\frac{2 G_{02} G_{03}}{r_{1}^{4}}\right] \\
L_{2}= & \left(4 W_{2} W_{5}-\frac{G_{03}^{2}}{r_{1}^{4}}\right) \Psi_{\mathrm{Gb}}^{2} \\
& +\left[4\left(W_{2} W_{3}+W_{0} W_{5}\right)-\frac{2 G_{03}}{r_{1}^{2}}-\frac{2 G_{00} G_{03}}{r_{1}^{4}}\right] \Psi_{\mathrm{Gb}} \\
& +\left(4 W_{0} W_{3}-\phi_{f p}^{2}-\frac{2 G_{00}}{r_{1}^{2}}-\frac{G_{00}^{2}}{r_{1}^{4}}\right) \\
W_{0}= & \frac{1}{2 \sinh \left(r_{1} L\right)}\left\{-H_{0} e^{-r_{1} L}+H_{3}+\frac{G_{00}}{r_{1}^{2}}\left(1-e^{-r_{1} L}\right)\right\} \\
W_{1}= & \frac{1}{2 \sinh \left(r_{1} L\right)}\left\{-H_{1} e^{-r_{1} L}+H_{4}+\frac{G_{02}}{r_{1}^{2}}\left(1-e^{-r_{1} L}\right)\right\} \\
W_{2}= & \frac{1}{2 \sinh \left(r_{1} L\right)}\left\{-H_{2} e^{-r_{1} L}+H_{5}+\frac{G_{03}}{r_{1}^{2}}\left(1-e^{-r_{1} L}\right)\right\} \\
W_{3}= & \frac{1}{2 \sinh \left(r_{1} L\right)}\left\{H_{0} e^{r_{1} L}-H_{3}-\frac{G_{00}}{r_{1}^{2}}\left(1-e^{r_{1} L}\right)\right\} \\
W_{4}= & \frac{1}{2 \sinh \left(r_{1} L\right)}\left\{H_{1} e^{r_{1} L}-H_{4}-\frac{G_{02}}{r_{1}^{2}}\left(1-e^{r_{1} L}\right)\right\} \\
W_{5}= & \frac{1}{2 \sinh \left(r_{1} L\right)}\left\{H_{2} e^{r_{1} L}-H_{5}-\frac{G_{03}}{r_{1}^{2}}\left(1-e^{r_{1} L}\right)\right\} .
\end{aligned}
$$




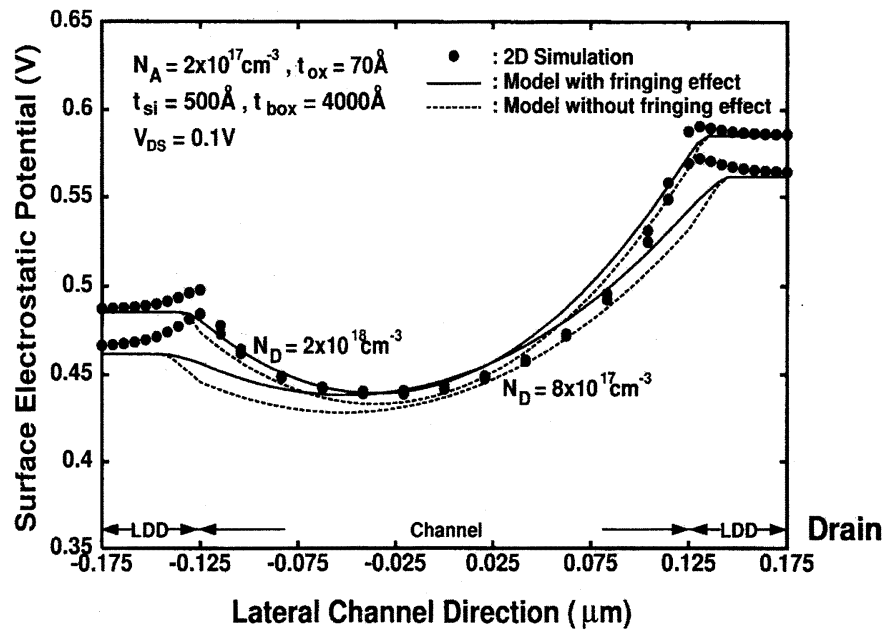

Fig. 3. Surface electrostatic potential at the front gate oxide/thin-film interface in the lateral channel region of the FD SOI nMOS device with the parameters as described in the caption of Fig. 2, for the doping densities of $2 \times 10^{18} \mathrm{~cm}^{-3}$ and $8 \times 10^{17} \mathrm{~cm}^{-3}$ in the n-LDD region, with its gate biased at the threshold voltage and its drain at $V_{\mathrm{DS}}=0.1 \mathrm{~V}$, based on the model and 2-D simulation results.

\section{Model Evaluation AND Discussion}

In order to assess the validity of the analytical model considering the fringing electric field effect from the sidewall oxide spacer, the analytical model results have been compared with the 2-D simulation results. Fig. 3 shows the surface electrostatic potential at the front gate oxide/thin-film interface in the lateral channel region of the FD SOI nMOS device with the parameters as described in the caption of Fig. 1, for the doping densities of $2 \times 10^{18} \mathrm{~cm}^{-3}$ and $8 \times 10^{17} \mathrm{~cm}^{-3}$ in the n-LDD region, with its gate biased at the threshold voltage $-V_{\mathrm{GS}}=$ $0.193 \mathrm{~V}$ and $V_{\mathrm{DS}}=0.1 \mathrm{~V}$, based on the model and the 2-D simulation results. As shown in the figure, at a lower n-LDD doping density of $8 \times 10^{17} \mathrm{~cm}^{-3}$, the depletion region of the n-LDD region $\left(L_{s}, L_{d}\right)$ increases. Therefore, the voltage drop in the n-LDD depletion region becomes larger-the effective drain voltage decreases, which leads to a lower minimum surface electrostatic potential in the channel. For the model results considering the fringing electric field effect at the interface between the n-LDD depletion region and the sidewall oxide spacer, extra flux exists-effective charge increases in the n-LDD depletion region, which can be understood from Gauss law. As a result, the voltage drop in the n-LDD depletion region decreases, which results in a higher effective drain voltage in the channel region as compared to the case without considering the fringing electric field effect. Therefore, considering the fringing electric field effect, the minimum surface electrostatic potential in the channel is higher-a lower threshold voltage. The fringing electric field effect gets stronger for the case with a lower doping density in the n-LDD region.

Fig. 4 shows the threshold voltage versus the channel length of the FD SOI nMOS device with the parameters as described in the caption of Fig. 1 with various thin-film doping densities, gate oxide thicknesses, and n-LDD doping densities, biased at $V_{\mathrm{DS}}=0.1 \mathrm{~V}$. Also shown in the figure are the results for the FD SOI nMOS with a front gate oxide of $40 \AA$ and a thin-film doping density of $3 \times 10^{17} \mathrm{~cm}^{-3}$. As shown in Fig. 4, for both the front gate oxide of 40 and $70 \AA$ cases, considering the fringing electric field effect, the threshold voltage is smaller. This can be understood using the reasoning as for Fig. 3. As shown in the figure, when the doping density of the n-LDD region becomes smaller, the reduction in the threshold voltage due to the fringing electric field effect becomes stronger. The reduction of the threshold voltage due to

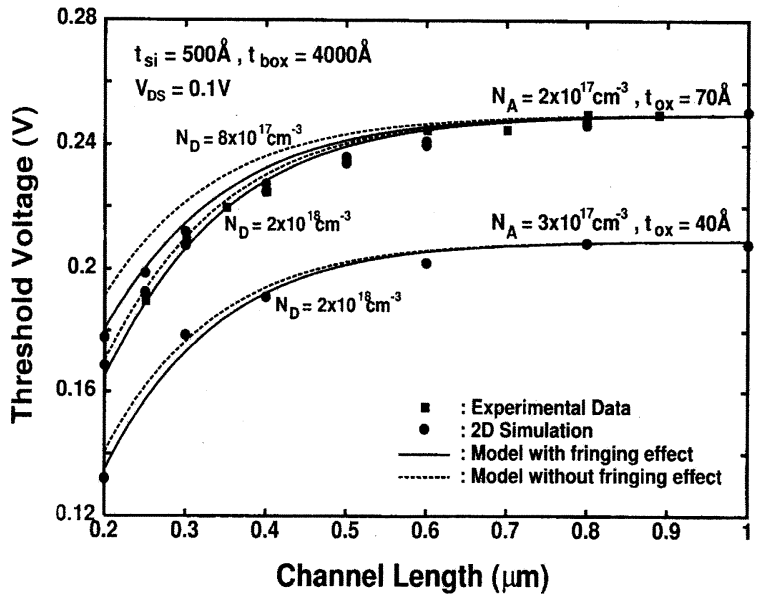

Fig. 4. Threshold voltage versus channel length of the FD SOI nMOS device with various thin-film doping densities, gate oxide thicknesses and n-LDD doping densities, biased at $V_{\mathrm{DS}}=0.1 \mathrm{~V}$ based on the experimental data (solid square), the 2-D simulation results (solid circle), and the model results considering the fringing electric field effect (solid line) and without (dashed line).

the fringing electric field effect is also dependent on the doping density of the $\mathrm{n}-\mathrm{LDD}$ region. Considering the fringing electric field effect, for the device with a front gate oxide of $70 \AA$, at a channel length of $0.2 \mu \mathrm{m}$ and with an n-LDD doping density of $2 \times 10^{18} \mathrm{~cm}^{-3}$, the threshold voltage is reduced by $5 \mathrm{mV}$ as compared to the case without the fringing electric field effect. When the n-LDD doping density is reduced to $8 \times 10^{17} \mathrm{~cm}^{-3}$, it is reduced by $10 \mathrm{mV}$, which is due to the increased fringing electric field effect in the widened depleted n-LDD region. As shown in the figure, for a channel length of $0.2 \mu \mathrm{m}$, with a front gate oxide of $40 \AA$ (Note that when the gate oxide thickness is scaled down from 70 to $40 \AA$, the thin-film doping density of the device is raised accordingly from $2 \times 10^{17} \mathrm{~cm}^{-3}$ to $3 \times 10^{17} \mathrm{~cm}^{-3}$ based on the scaling law), for the device with the n-LDD doping density of $2 \times 10^{18} \mathrm{~cm}^{-3}$, the threshold voltage considering the fringing electric field effect becomes smaller by $5.6 \mathrm{mV}$ as compared to the case without considering the fringing electric field, which is larger than the case with a gate oxide thickness of $70 \AA-5 \mathrm{mV}$. Therefore, with the fringing electric field effect, the threshold voltage is reduced. When the FD SOI nMOS device is scaled down with a thinner gate oxide, the fringing electric field effect on the short-channel effect threshold voltage via the $\mathrm{LDD} /$ sidewall oxide spacer structure is increased.

\section{CONCLUSION}

In this paper, analysis of the fringing electric field effect on the threshold voltage of FD SOI nMOS devices with the LDD/sidewall oxide spacer structure based on a closed-form analytical model derived from the 2-D Poisson's equation and using the conformal mapping technique has been reported. Based on the analytical model, as verified by the experimental data and the 2-D simulation results, with a lower n-LDD doping density, the fringing electric field effect in the sidewall oxide spacer lowers the short-channel effect.

\section{REFERENCES}

[1] J. B. Kuo and S. C. Lin, Low-Voltage SOI CMOS VLSI Devices and Circuits. New York: Wiley, 2001.

[2] J. B. Kuo and K. W. Su, CMOS VLSI Engineering: Silicon On Insulator (SOI). Boston, MA: Kluwer, 1998.

[3] J. Chen, S. Parke, K. King, F. Assaderaghi, P. K. Ko, and C. Hu, "A high speed SOI technology with $12 \mathrm{ps} / 18$ ps gate delay operating at $5 \mathrm{~V} / 1.5 \mathrm{~V}$.," in IEDM Tech. Dig., 1992, pp. 35-38. 
[4] Y. Yamaguchi, T. Iwamatsu, H.-O. Joachim, H. Oda, Y. Inoue, T. Nishimura, and K. Tsukamoto, "Source-to-drain breakdown voltage improvement in ultrathin-film SOI MOSFETs using a gate-overlapped LDD structure," IEEE Trans. Electron Dev., vol. 41, pp. 1222-1226, July 1994.

[5] Y. Omura, S. Nakashima, K. Izumi, and T. Ishii, "0.1-um-gate, ultrathin-film CMOS devices using SIMOX substrate with 80-nm-thick buried oxide layer," in IEDM Tech. Dig., 1991, pp. 675-678.

[6] Y. Omura and K. Izumi, "Physical background of substrate current characteristics and hot-carrier immunity in short-channel ultrathin-film MOSFETs/SIMOX," IEEE Trans. Electron Devices, vol. 41, pp. 352-358, Mar. 1994.

[7] G. C. F. Yeap, S. Krishnan, and M. R. Lin, "Fringing-Induced Barrier Lowering (FIBL) in sub-100nm MOSFETs with high-K gate dielectrics," Electronics Letters, vol. 34, pp. 1150-1152, May 1998.

[8] S.-C. Lin, J. B. Kuo, K. -T. Huang, and S.-W. Sun, "A closed-form backgate-bias related inverse narrow-channel effect model for deep-submicron VLSI CMOS devices using shallow trench isolation," IEEE Trans. Electron Devices, vol. 47, pp. 725-733, Apr. 2000.

[9] MEDICI, Two-Dimensional Device Simulation Program. Sunnyvale, CA: Technology Modeling Associates, 1996.

[10] J. B. Kuo, K. H. Yuan, and S. C. Lin, "Compact threshold-voltage model for short-channel partially depleted (PD) SOI dynamic-threshold MOS (DTMOS) devices," IEEE Trans. Electron Devices, vol. 49, pp. 190-196, Jan. 2002.

[11] I. N. Senddon, The Use of Integral Transforms. New York: McGrawHill, 1972.

[12] S. S. Chen and J. B. Kuo, "Deep submicrometer double-gate fully-depleted SOI PMOS devices: A concise short-channel effect threshold voltage model using a quasi-2-D approach," IEEE Trans. Electron Devices, vol. 43, pp. 1387-1393, Sept. 1996.

\section{On the Evaluation of Performance Parameters of MOSFETs With Alternative Gate Dielectrics}

Khaled Z. Ahmed, Philip A. Kraus, Chris Olsen, and Faran Nouri

\begin{abstract}
This brief discusses a benchmarking methodology for the evaluation of performance parameters $\left(g_{\operatorname{mmax}}\right.$ and $\left.I_{\mathrm{dsat}}\right)$ of MOSFETs with alternative gate dielectrics. It is shown that assuming ideal scaling for either $g_{\operatorname{mmax}}$ or $I_{\mathrm{dsat}}$ with electrical oxide thickness $\left(g_{\operatorname{mmax}}\right.$ or $I_{\text {dsat }} \propto T_{\text {oxinv }}{ }^{-\alpha}$ with $\alpha=1$ ) instead of using experimental scaling trends for a baseline dielectric results in unrealistically pessimistic conclusions about the performance of alternative gate dielectrics. Factors in addition to mobility reduction which can contribute to sub-ideal scaling $(\alpha<1)$ for any dielectric are discussed. This benchmarking methodology for performance evaluation is demonstrated for oxynitride gate dielectric films with equivalent oxide thickness (EOT) approaching 11 A.
\end{abstract}

Index Terms-Drive current, high- $\kappa$, MOSFET, oxynitrides, transconductance, ultrathin oxides.

\section{INTRODUCTION}

Scaling of high-performance MOSFETs below the 100-nm technology node requires the use of gate dielectrics with equivalent oxide thickness (EOT) thinner than $15 \AA$ [1]. Scaling $\mathrm{SiO}_{2}$ to this thickness range is limited by high gate tunneling current. Alternative materials that have dielectric constant higher than that of $\mathrm{SiO}_{2}$ may enable

Manuscript received June 18, 2003; revised August 18, 2003. The review of this brief was arranged by Editor C.-Y. Lu.

The authors are with the Front End Products Group, Applied Materials, Inc., Santa Clara, CA 95054 USA (e-mail: khaled_ahmed@amat.com).

Digital Object Identifier 10.1109/TED.2003.81925
CMOS scaling requirements of small EOT and acceptable gate leakage current [1]. These materials include oxynitrides with high nitrogen content $(3.9<\kappa<7.5)$, and metal oxides such as $\mathrm{HfO}_{2}(20<\kappa<$ 30 ). Among other process and integration problems, mobility (performance) reduction is particularly challenging to using these materials for high performance CMOS applications [1]. In developing these dielectrics, it is common to compare their performance against baseline or benchmark dielectrics (pure or lightly nitrided thermal $\mathrm{SiO}_{2}$ ) that have effective mobility near the universal limit.

In many cases, the evaluation of the performance of alternative gate dielectrics is based on the maximum linear transconductance $\left(g_{\operatorname{mmax}}\right)$ and drive current $\left(I_{\mathrm{dsat}}\right)$ of long channel devices as an alternative to comparing effective mobility ( $\mu_{\text {eff }}$ ) [2]-[5] . This is usually done for practical reasons where fast and statistically sound data can be measured using automatic wafer-level testers. In order to normalize for differences in oxide thickness, such an evaluation assumes the standard scaling relationships for $g_{\operatorname{mmax}}$ and $I_{\mathrm{dsat}}$ with $T_{\text {oxinv }}$, i.e.,

$$
\begin{aligned}
g_{m \max } & \propto T_{\text {oxinv }}^{-1} \\
I_{\text {dsat }} & \propto T_{\text {oxinv }}^{-1} .
\end{aligned}
$$

In this case, the performance of an alternative gate dielectric against a benchmark dielectric is evaluated by comparing $g_{\operatorname{mmax}} \times T_{\text {oxinv }}$ and $I_{\text {dsat }} \times T_{\text {oxinv }}$.

The validity of (1) is based on the simplifying assumptions of (3) and (4), and the validity of (2) is based on the simplifying assumption given by (4) and (5) [6]

$$
\begin{aligned}
Q_{i} \frac{\partial \mu_{\mathrm{eff}}}{\partial V_{\mathrm{g}}} & \ll \mu_{\mathrm{eff}} \frac{\partial Q_{i}}{\partial V_{\mathrm{g}}} \\
\mu_{\mathrm{eff}} & \neq f\left(T_{\text {oxinv }}\right) \\
\mu_{\mathrm{eff}} & \neq f\left(\frac{\left(V_{\mathrm{g}}-V_{\mathrm{T}}\right)}{T_{\text {oxinv }}}\right) .
\end{aligned}
$$

The assumptions given by (3)-(5) may not hold for aggressively scaled oxides as evidenced by experimental data [2], [7]-[9]. The observed experimental dependence of $\mu_{\mathrm{eff}}$ on $V_{\mathrm{g}}(3)$ at low fields will result in a slight deviation from (1). The observed experimental dependence of $\mu_{\text {eff }}$ on $T_{\text {oxinv }}(4)$ has been related to a variety of scattering mechanisms. Scattering mechanisms that can be affected by process parameters include Coloumbic scattering due to fixed and interfacial oxide charges or ionized channel impurities $\left(\mu_{\mathrm{C}}\right)$, and interface roughness at the oxide/channel interface $\left(\mu_{\mathrm{SR}}\right)$ [8]. Scattering mechanisms that are due to inevitable physical mechanisms include remote charge scattering (RCS) due to charge impurities in the gate material [10], [11]. The RCS mobility ( $\left.\mu_{\mathrm{RCS}}\right)$ has a strong dependence on physical oxide thickness, significantly degrading mobility for oxides less than $20 \AA$ [10], [11]. The effect of remote charge scattering on the scaling of $g_{\text {mmax }}$ with $T_{\text {oxinv }}$ is demonstrated in the simulation results of Fig. 1, where inclusion of the RCS effect results in sub-ideal scaling of the form $g_{m \text { max }} \propto T_{\text {oxinv }}^{-\alpha}$ with $\alpha<1$. It can be seen that $\alpha$ is dependent on the theoretical model used to estimate the RCS mobility component for given channel and polysilicon gate doping densities.

The universal experimental dependence of $\mu_{\mathrm{eff}}$ on electric field $\left(E=1 / \varepsilon_{\mathrm{Si}}\left(Q_{\mathrm{B}}+1 / \eta Q_{i}\right) \approx\left(V_{\mathrm{g}}-V_{\mathrm{T}}\right) / 6 T_{\text {oxinv }}\right)$ was shown to result in smaller $\mu_{\text {eff }}$ (at constant $V_{\mathrm{g}}-V_{\mathrm{T}}$ ) for thinner $T_{\text {oxinv }}$ [12], [13]. This dependence results in subideal scaling of $I_{\mathrm{dsat}}$ with $T_{\text {oxinv }}$ (i.e., $I_{\text {dsat }} \propto T_{\text {oxinv }}^{-\beta}$ with $\beta=0.8$ ) [12], [13]. The value of $\beta$ can be reduced further if process parameters were not optimized to maintain the same $\mu_{\mathrm{SR}}$ for thin and thick oxides.

Based on the above discussion, it is postulated that simple semi-empirical power laws may be used to describe the scaling of $g_{\operatorname{mmax}}$ and 